\title{
Receptive-Field Subfields of V2 Neurons in Macaque Monkeys Are Adult-Like Near Birth
}

\author{
Bin Zhang, ${ }^{1,2}$ Xiaofeng Tao, ${ }^{1}$ Guofu Shen, ${ }^{1}$ Earl L. Smith III, ${ }^{1}$ Izumi Ohzawa, ${ }^{3}$ and Yuzo M. Chino ${ }^{1}$ \\ ${ }^{1}$ College of Optometry, University of Houston, Houston, Texas 77204-2020, ${ }^{2}$ College of Optometry, NOVA Southeastern University, Fort Lauderdale, Florida \\ 33325, and ${ }^{3}$ Graduate School of Frontier Science, Osaka University, Osaka 560-8531, Japan
}

Infant primates can discriminate texture-defined form despite their relatively low visual acuity. The neuronal mechanisms underlying this remarkable visual capacity of infants have not been studied in nonhuman primates. Since many V2 neurons in adult monkeys can extract the local features in complex stimuli that are required for form vision, we used two-dimensional dynamic noise stimuli and local spectral reverse correlation to measure whether the spatial map of receptive-field subfields in individual V2 neurons is sufficiently mature near birth to capture local features. As in adults, most V2 neurons in 4-week-old monkeys showed a relatively high degree of homogeneity in the spatial matrix of facilitatory subfields. However, $\sim 25 \%$ of V2 neurons had the subfield map where the neighboring facilitatory subfields substantially differed in their preferred orientations and spatial frequencies. Over $80 \%$ of V 2 neurons in both infants and adults had "tuned" suppressive profiles in their subfield maps that could alter the tuning properties of facilitatory profiles. The differences in the preferred orientations between facilitatory and suppressive profiles were relatively large but extended over a broad range. Response immaturities in infants were mild; the overall strength of facilitatory subfield responses was lower than that in adults, and the optimal correlation delay ("latency") was longer in 4-week-old infants. These results suggest that as early as 4 weeks of age, the spatial receptive-field structure of V2 neurons is as complex as in adults and the ability of V2 neurons to compare local features of neighboring stimulus elements is nearly adult like.

\section{Introduction}

Recognition of an object in visual scenes requires the ability to extract visual form cues that vary considerably in complexity. While low-level spatial vision depends on the identification of forms defined by differences in luminance cues, complex global form vision depends on perceptual grouping of local features over an extended region of space. For example, the sensitivity to texture-defined form is influenced by the ability to extract the appropriate image properties, to integrate these properties, and to segment the shape that is represented (Geisler et al., 2001; Geisler, 2008; El-Shamayleh et al., 2010; Ing et al., 2010; ElShamayleh and Movshon, 2011).

Newborn human and nonhuman primates have limited visual capacities. However, newborn human infants are capable of discriminating the orientation of luminance-defined contours of low spatial frequencies near birth (Atkinson et al., 1988). Infant macaque monkeys can discriminate texture- or contrast-defined form as early as 6-8 weeks of age (El-Shamayleh et al., 2010).

\footnotetext{
Received Sept. 13, 2012; revised Dec. 10, 2012; accepted Dec. 14, 2012.

Author contributions: B.Z., E.L.S., and Y.M.C. designed research; B.Z., X.T., G.S., and Y.M.C. performed research; B.Z., I.O., and Y.M.C. contributed unpublished reagents/analytic tools; B.Z., X.T., G.S., I.O., and Y.M.C. analyzed data; B.Z. and Y.M.C. wrote the paper.

This research was supported by NIH/National Eye Institute Research Grants EY-008128 (Y.C.) and EY-003611 (E.L.S.); Core Grant P30 EY-007551; and Ministry of Education, Culture, Sports, Science, and Technology of Japan Kakenhi Grants 22135006 and 223001 (I.0.).

The authors declare no conflicting financial interests.

Correspondence should be addressed to Dr. Yuzo M. Chino, College of Optometry, University of Houston, 505 J Davis Armistead Building, Houston, TX 77204-2020. E-mail: ychino@uh.edu.

DOI:10.1523/JNEUROSCI.4377-12.2013

Copyright $\odot 2013$ the authors $\quad 0270-6474 / 13 / 332639-11 \$ 15.00 / 0$
}

Human infants also perform well in similar visual tasks near birth (Hou et al., 2003; Norcia et al., 2005; Sireteanu et al., 2005).

In adult monkeys, neurons in extrastriate visual area are thought to act as "integrators" of local stimulus information that is processed by V1 in a variety of global perceptual phenomena. Because of the convergence of primarily feedforward and local signals and the progressively larger receptive-field (RF) sizes of neurons in higher-order visual areas, V2 and V4 neurons become sensitive to angled contours that make up critical aspects of global shape and, consequently, become capable of efficiently linking local feature information (Pasupathy and Connor, 2002; Ito and Komatsu, 2004; Anzai et al., 2007; Willmore et al., 2010; ElShamayleh and Movshon, 2011).

Developmentally, although the spatiotemporal filter properties of V2 neurons mature rapidly after birth (Zheng et al., 2007; Maruko et al., 2008), we do not know whether the receptive-field structure of V2 neurons in infant primates is organized in a manner that would allow them to encode more complex stimulus features that are composed of different orientations and spatial frequencies. Therefore, we used dynamic two-dimensional noise stimuli and the local spectral reverse correlation (LSRC) method to study the maturation of the spatial matrix of subfields in V2 neurons (Nishimoto et al., 2006; Tao et al., 2012). The LSRC method is quite effective in revealing response profiles that contain local variations in orientation and spatial frequency (SF) tuning properties. It is based on spectral analysis in the twodimensional spatial frequency domain for spatially localized areas within and around the receptive field. The advantages of using LSRC are that, unlike conventional methods (Gallant et al., 1993, 
1996; Pasupathy and Connor, 2001, 2002; Anzai et al., 2007), LSRC has stimulus sets with infinite possible configurations, makes minimum assumptions about receptive subfield organization, is applicable for all cell types, and can visualize suppressive as well as facilitatory profiles. We will show that the spatial receptive-field structure of V2 neurons is as complex at 4 weeks of age as in adults.

\section{Materials and Methods}

The subjects were five 4-week-old, four 8-week-old, and six adult monkeys (Macaca mulatta). Seven were males, and eight were females. The weights of the infant monkeys varied between 450 and $600 \mathrm{~g}$ at 4 weeks and between 400 and $960 \mathrm{~g}$ at 8 weeks. All experimental and animal care procedures were in compliance with the Guiding Principles for Research Involving Animals and were approved by the Institutional Animal Care and Use Committee of the University of Houston.

\section{Surgical preparation}

Microelectrode recording experiments were conducted in anesthetized and paralyzed monkeys. The surgical preparation methods have been described previously (Maruko et al., 2008; Tao et al., 2012). Briefly, the animals were initially anesthetized with an intramuscular injection of ketamine hydrochloride $(20-30 \mathrm{mg} / \mathrm{kg})$ and acepromazine malerate $(0.15-0.2 \mathrm{mg} / \mathrm{kg})$. A venous cannula was placed to allow subsequent continuous infusion of propofol $(4 \mathrm{mg} / \mathrm{kg} / \mathrm{h})$ and sufentanyl citrate $(0.05-010 \mu \mathrm{g} / \mathrm{kg} / \mathrm{h})$. A tracheotomy was performed to facilitate artificial ventilation with a mixture of $59 \% \mathrm{~N}_{2} \mathrm{O}, 39 \% \mathrm{O}_{2}$, and $2 \% \mathrm{CO}_{2}$. To insert the electrode, a craniotomy and a small durotomy were performed over the lunate sulcus, through which a guide tube was positioned close to the surface. The exposed area was protected with a plastic well that was filled with warm agar and then closed with melted wax. The animals were paralyzed with an intravenous injection of vecuronium bromide (Norcuron; $0.1 \mathrm{mg} / \mathrm{kg} / \mathrm{h}$ ) after all surgical procedures were completed. Core body temperature was monitored and kept at $37.6^{\circ} \mathrm{C}$ by a homeothermic controller (Harvard Apparatus). Cycloplegia was produced, and the animals' corneas were protected with rigid gas-permeable, extended-wear contact lenses. Retinoscopy was used to determine the contact lens parameters required to focus the eyes on the stimulus screens.

\section{Recording and visual stimulation}

Electrode penetrations were confined to a parasagittal plane, and the angle of the penetration was typically $15^{\circ}$ from vertical. The penetrations were started right behind the blood vessels running along the lunate sulcus and $12-15 \mathrm{~mm}$ lateral to the midline. Unit activity was isolated at an interval of 50-100 $\mu \mathrm{m}$ steps. The penetrations ended when the electrode exited V2. For each penetration, we made records of the cortical depth for each isolated unit. This approach allowed us to sample units consistently from similar regions of V2 for both infants and adults. All receptive fields both in infants and adults were located within $5^{\circ}$ of the center of the projected fovea.

A monitor (Vision Research Graphics) with ultra-short persistence (frame rate $=140 \mathrm{~Hz}, 800 \times 600$ pixels) was used to present visual stimuli. The viewing distance was set to $114 \mathrm{~cm}$ where the display subtended a visual angle of $20^{\circ}$ (horizontal) $\times 15^{\circ}$ (vertical). Multiunit or single-unit activities were extracellularly recorded with tungsten-in-glass microelectrodes (Fredric-Haer). Action potentials from individual cortical neurons were amplified, isolated, and stored using the data acquisition components in our workstation (Tucker-Davis Technology). For each isolated neuron, handheld stimuli were initially used to map the receptive fields in each eye and to qualitatively determine the neuron's ocular dominance and preferred stimulus orientation. Quantitative measurements using drifting gratings followed these procedures and are described below.

Measurements with drifting sine wave gratings. A neuron's responses to drifting gratings were sampled at a rate of $140 \mathrm{~Hz}$ (7.14 ms bin widths) and compiled into peristimulus time histograms (PSTHs) that were equal in duration to, and synchronized with, the temporal cycle of the grating. The amplitude and phase of the temporal response components in the PSTHs were determined by Fourier analysis. The stimuli were

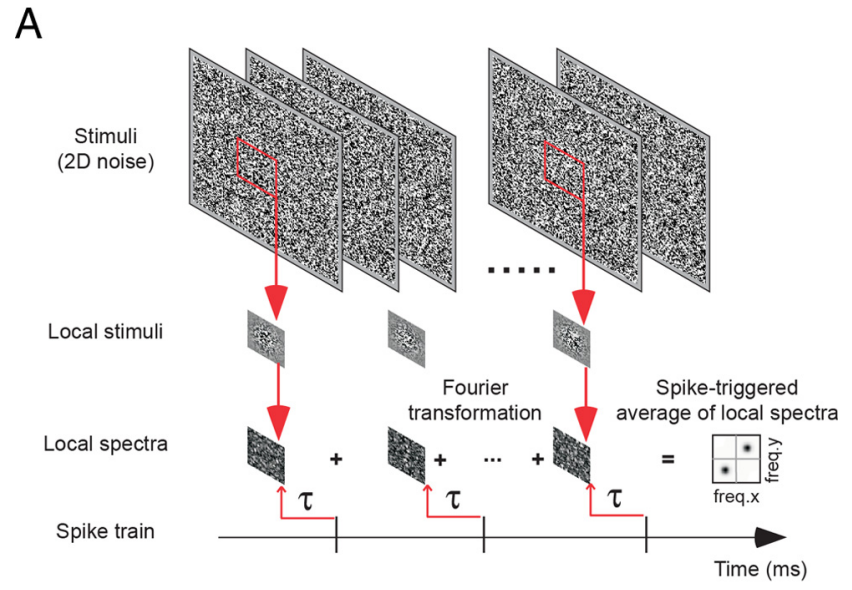

B

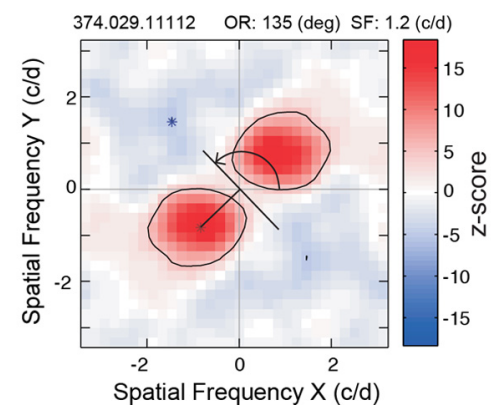

Figure 1. Schematic diagram of the LSRC analysis (see Materials and Methods for details). $\boldsymbol{A}$ The visual stimuli and analysis procedure used to derive LSRC maps. We calculated a crosscorrelation between the spike train and the amplitude spectra of Gaussian-windowed stimuli to obtain a two-dimensional frequency tuning function for the given subfield. $\boldsymbol{B}$, An example of the spike-triggered average of local spectra (local spectral selectivity map or subfield). The $x$ and $y$-axes show vertical and horizontal spatial frequency in cycle/degree (c/d). The facilitations and suppressions are indicated by red and blue, respectively. Asterisks show the location of the highest and lowest z-scores that correspond to the frequency of the maximum facilitation and suppression, respectively. The scale bar with $z$-scores is illustrated on the right. The distance from the origin to the peak of the excitation indicated the optimal spatial frequency for the local subfield of the receptive field. The angle perpendicular to the line connecting the origin and the excitation peak (with the horizontal axis) depicted the optimal orientation for the local subfield (curved arrow).

presented to the right or left eye in a randomly ordered sequence for relatively short periods (e.g., $3.22 \mathrm{~s}$ ). Rerandomized stimulus sequences were repeated three to six times during a given experiment. Blank stimuli (i.e., zero contrast control) were included in each repeat to provide a measure of the neuron's maintained firing rate. For each neuron, the orientation, spatial frequency, and size-tuning functions were first obtained using sine wave gratings ( $80 \%$ contrast and temporal frequency $=$ $3.1 \mathrm{~Hz})$.

The preferred orientation and orientation bandwidth for each receptive field were determined by fitting the orientation tuning functions with wrapped Gaussian functions (Swindale, 1998):

$$
\begin{gathered}
n=\propto \\
\left.G(\theta)=m_{1} \sum \exp -\left(\theta-m_{2}+180 n\right)^{2} /\left(2 m_{3}^{2}\right)\right] \\
n=-\propto
\end{gathered}
$$

where $\theta$ is orientation, $m_{1}$ is response amplitude, $m_{2}$ is preferred orientation, and $m_{3}$ is the SD of the Gaussian function.

Each cell's optimal spatial frequency was determined by fitting the response data with the following Gaussian functions (DeAngelis et al., 1993):

$$
G(f)=m_{1} \exp \left[-\left(f-f_{0}\right)^{2} /\left(2 s^{2}\right)\right],
$$


A
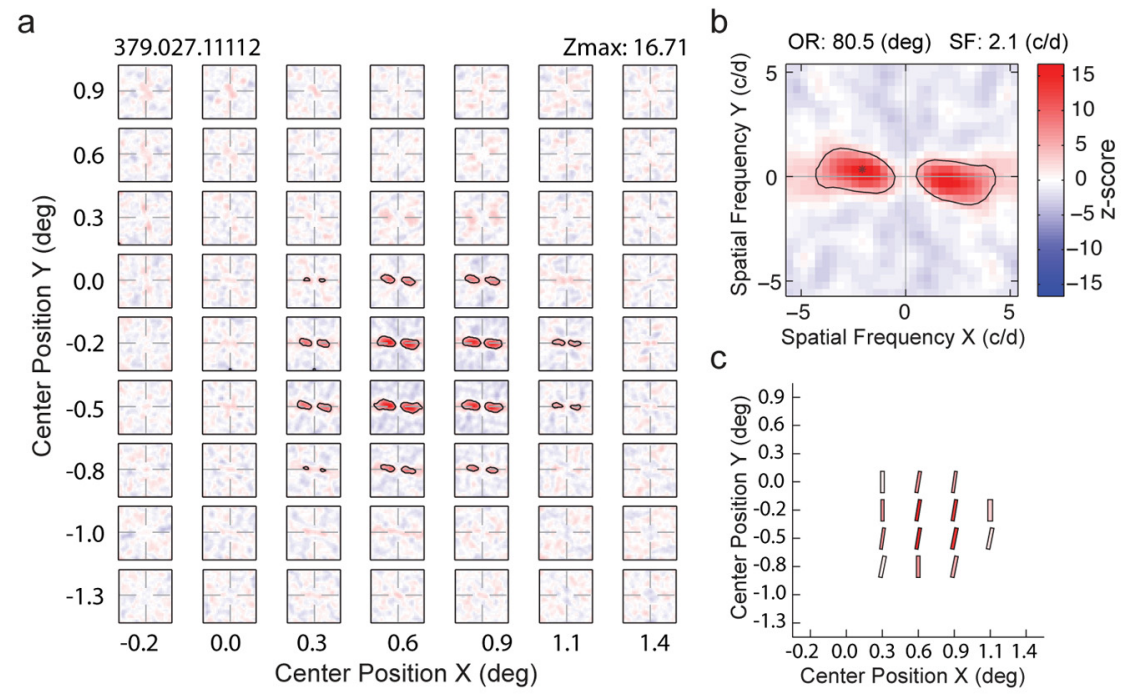

C

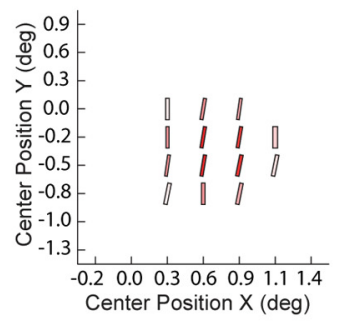

B

a

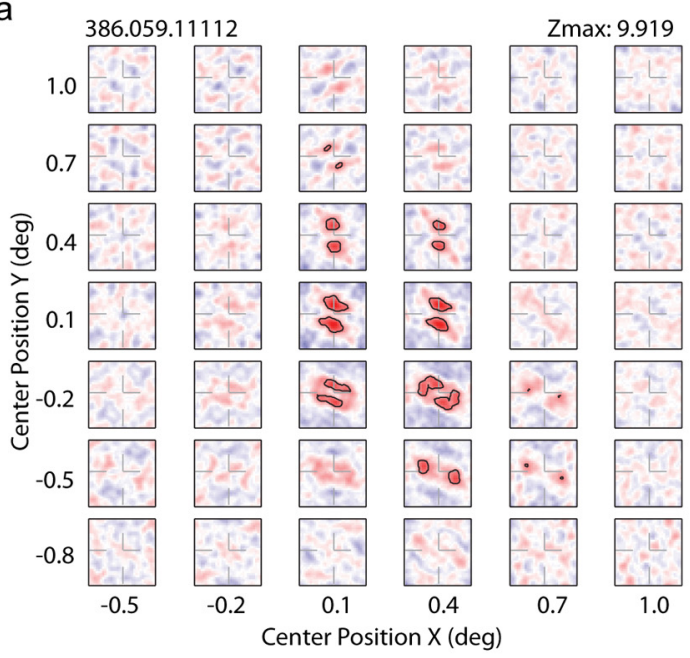

b

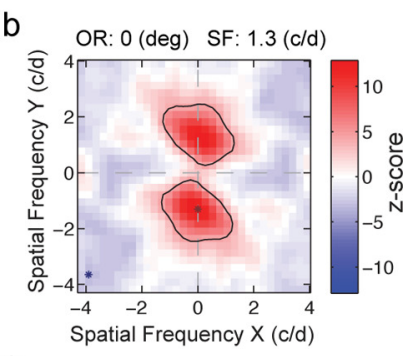

C

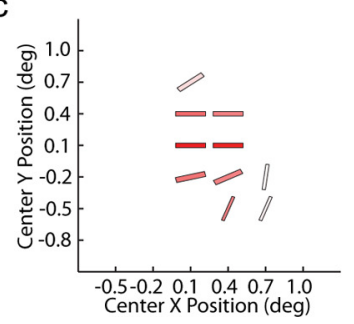

Figure 2. $\quad A a, B a, A$ spatial matrix of subfields with facilitatory profiles in a V2 neuron from an adult monkey that exhibited spatial homogeneity of orientation and spatial frequency within its receptive field $(\boldsymbol{A a})$ and an adult V2 neuron with highly heterogeneous subfield matrix $(\boldsymbol{B} \boldsymbol{a})$. $\boldsymbol{A} \boldsymbol{b}, \boldsymbol{B} \boldsymbol{b}$, Detail profile of the subfield with the maximum $z$-score. $\boldsymbol{A} \boldsymbol{c}, \boldsymbol{B} \boldsymbol{c}$, Schematic diagram showing the preferred orientation (bar angle) and spatial frequencies (width), and the maximum $z$-scores (saturation) of the subfields. c/d, Cycles per degree; deg, degree.

where $f$ is spatial frequency, $f_{0}$ is preferred spatial frequency, and $s$ is the SD of the Gaussian function.

To determine the position and extent of a neuron's receptive field center and surround and the strength of surround suppression, we measured area-summation functions with drifting high-contrast $(80 \%)$ sinusoidal gratings of optimized orientation, spatial frequency, and temporal frequency (Zhang et al., 2005). Specifically, the neuronal responses were measured as a function of the diameter of the grating patch. The measured area response functions were fitted by the following formula:

$$
R(x)=K_{c} L_{c}(x) /\left[1+K_{s} L_{s}(x)\right],
$$

where $L_{c}(x)=\left[w_{c} \times \operatorname{erf}\left(x / w_{c}\right)\right]^{2} ; L_{s}(x)=\left[w_{s} \times \operatorname{erf}\left(x / w_{s}\right)\right]^{2}$, erf is the error function, $x$ is the stimulus diameter, $K_{c}$ and $K_{s}$ are the gains of the center and surround, $L_{c}$ and $L_{s}$ are the summed squared activities of the center and surround mechanisms; $w_{c}$ and $w_{s}$ represented the spatial extents of the center and surround components; and $w_{c}$ is always constrained to be less than $w_{s}$ during our curve-fitting procedures.
The receptive field center size was determined by searching for the smallest center stimulus diameter at which neuronal discharges reached $95 \%$ of the peak firing rate. The boarder of the surround was the point at which further increases in stimulus diameter did not alter the response amplitude. The surround suppression strength was quantified for each neuron by calculating a suppression index: suppression index $=$ (peak response suppressed responses)/peak response for the unit's area summation function.

LSRC method. The details of visual stimulation and data analysis for the LSRC method have been described previously (Nishimoto et al., 2006; Tao et al., 2012). The control functions and the stimulus routines were performed using custom software on two Windows-based personal computers. A dynamic two-dimensional noise array $(51 \times 51$ elements $)$ that covers an area of three times larger in width and height (typical ranges were from $1^{\circ} \times 1^{\circ}$ to $12^{\circ} \times 12^{\circ}$ ) than the classical receptive field was used as the stimulus for each isolated V2 neuron. The luminance of each element in the noise array was bright $\left(99 \mathrm{~cd} / \mathrm{m}^{2}\right)$, dark $\left(1 \mathrm{~cd} / \mathrm{m}^{2}\right)$, or equal to the mean luminance of the display $\left(50 \mathrm{~cd} / \mathrm{m}^{2}\right)$. The noise array was redrawn with a new noise pattern every $28 \mathrm{~ms}$ (four video frames). Typically, 15 blocks of the noise arrays (a total of 62,565 frames) were presented to obtain a sufficient number of spikes for subsequent data analysis. This measurement took $\sim 30$ min for each neuron.

We calculated the spike-triggered average of the amplitude spectra produced by the noise array for a given subfield to obtain the subfield's two-dimensional frequency tuning function (Fig. $1 A$ ). The subfields were windowed by a twodimensional Gaussian function, and the frequency spectra were calculated by a standard fast Fourier transform algorithm with zero padding (Press et al., 1992). The center of the window was stepped typically by $1 \mathrm{SD}$ of the Gaussian function. A joint spatial frequency and orientation profile was obtained by interpreting the twodimensional frequency tuning plot as a polar coordinate representation. The distance from the origin to the peak of the excitation represented the optimal spatial frequency for the local subfield of the receptive field. The angle perpendicular to the line connecting the origin and the excitation peak (with the horizontal axis) represented the optimal orientation for the local subfield (Fig. 1B). By systematically changing the positions of the subfield for calculating the spectra, a spatial matrix of subfields was obtained. Therefore, the final matrix describes the tuning profile of the neuron as a function of position $(x, y)$ as well as spatial frequency and orientation in a joint manner. We optimized the number of positions/spacings for each unit depending on the spatial frequency tuning of the unit; for neurons with bandpass SF tuning profiles, the analysis window covered at least half of the period for the optimal spatial frequency within 1 SD of the Gaussian. In rare cases where neurons had low-pass SF tuning functions, we used the SD value corresponding to one-fifth of the mapped area.

We calculated spike-triggered averages of stimulus local spectra for correlation delays from 0 to $150 \mathrm{~ms}$ in $15 \mathrm{~ms}$ steps. Then, the optimal correlation delay was determined as the delay at which the maximal signal amplitude was found. Typical optimal correlation delays varied from 45 to $90 \mathrm{~ms}$. The average number of spikes per recording for our population of neurons was 4253 spikes for 4 -week-old, 4941 spikes for 8 -week-old, and 6565 spikes for adult monkeys, respectively. 
A a

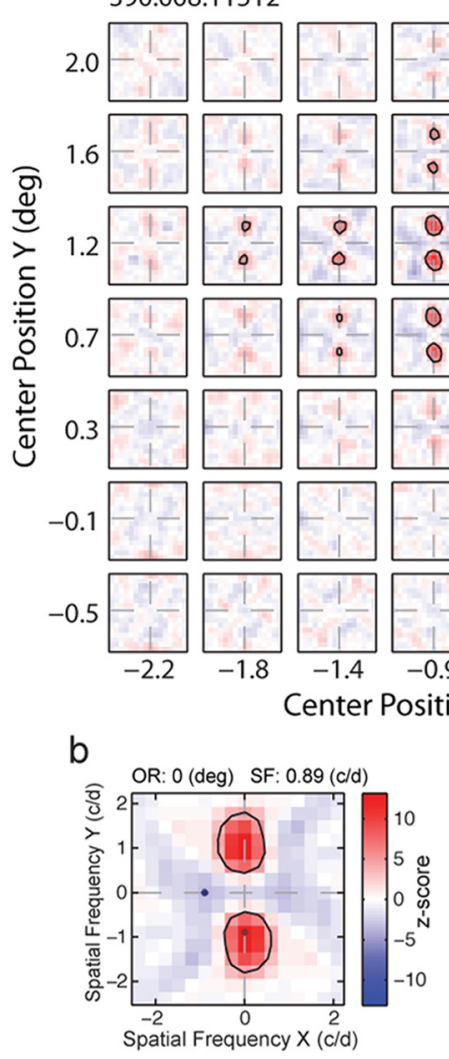

Zmax: 13.15

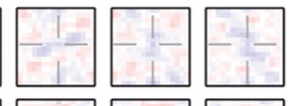

B

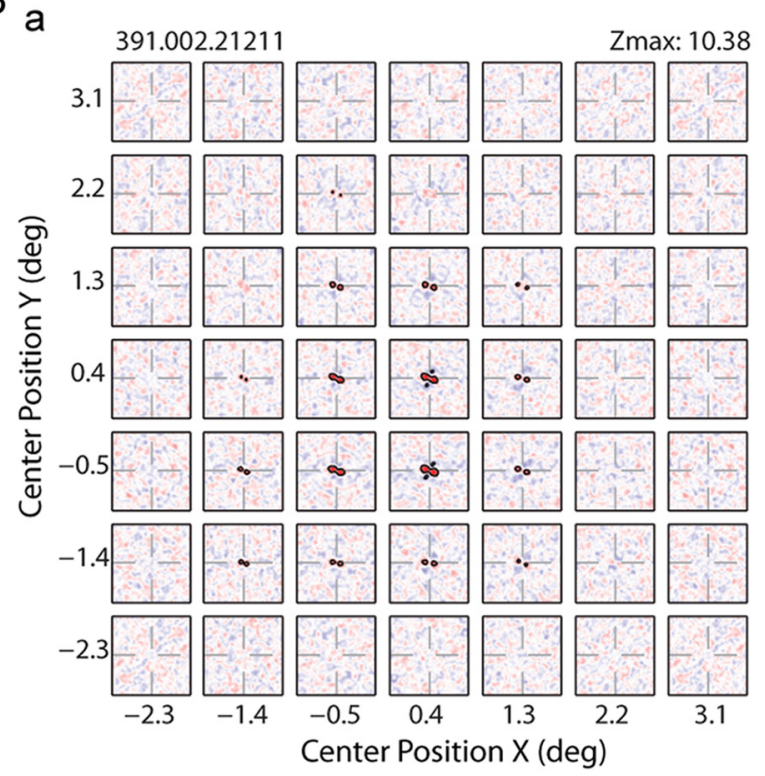

$c$

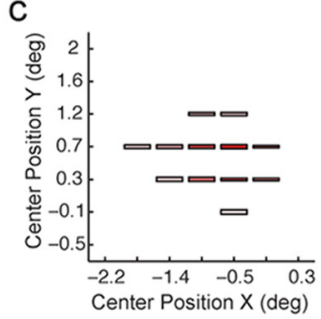

b

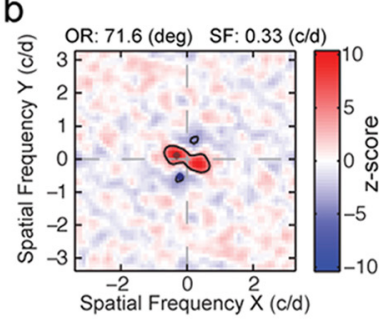

$c$
C

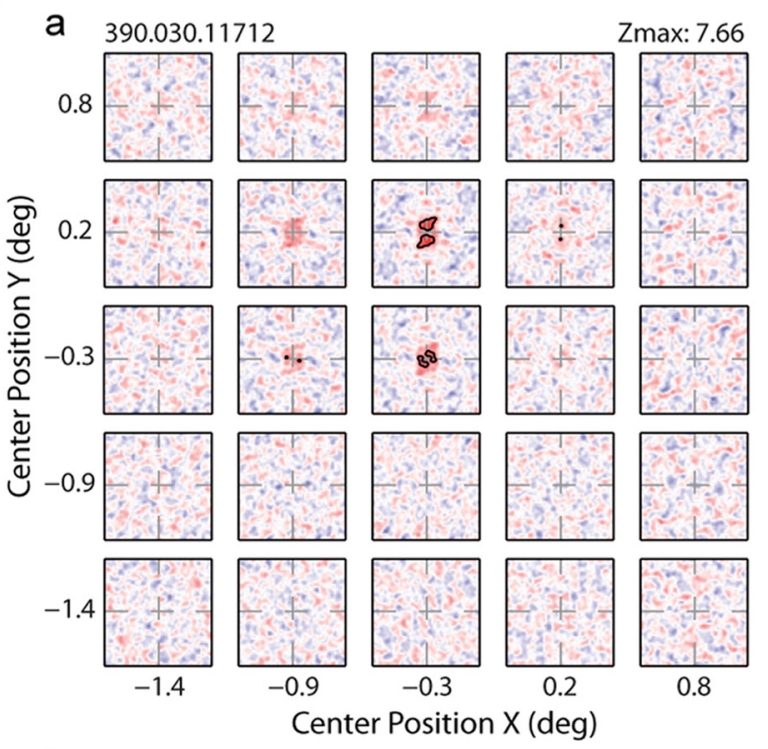

b

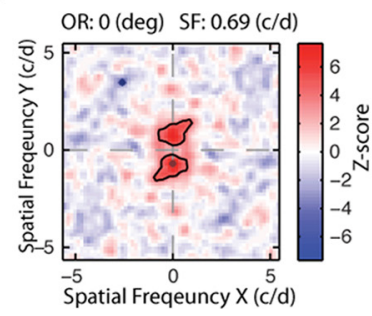

$c$

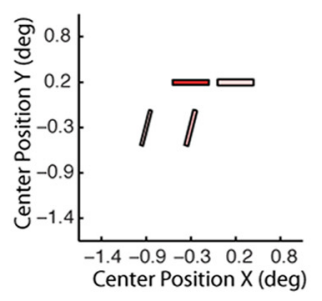

D

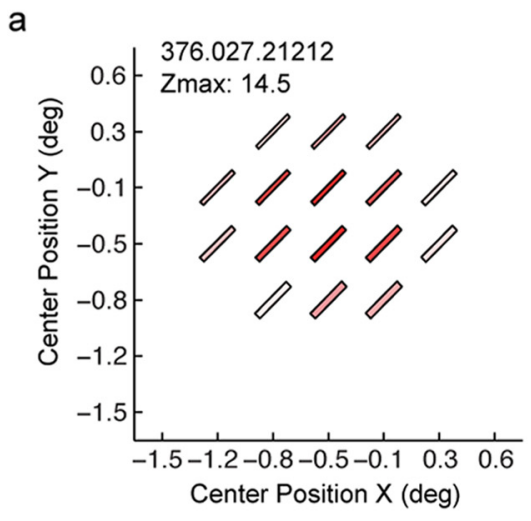

b

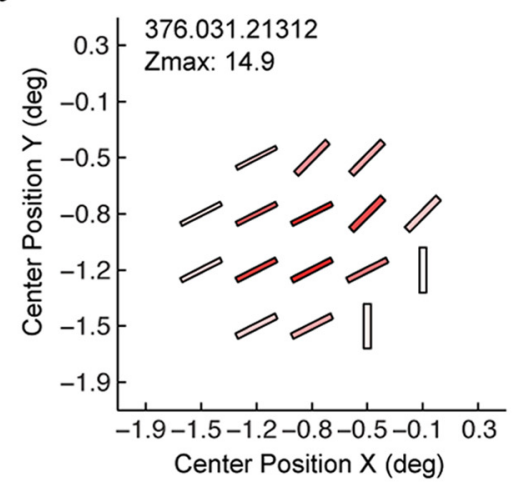

Figure 3. $A a, B a, C a, A$ spatial matrix of subfields with facilitatory profiles in a V2 neuron from a 4-week-old monkey that exhibited spatial homogeneity of orientation and spatial frequency within its receptive field $(\boldsymbol{A} \boldsymbol{a})$ and V2 neurons with heterogeneous subfield matrix from 4-week-old infants ( $\boldsymbol{B} \boldsymbol{a}, \boldsymbol{C} \boldsymbol{a})$. $\boldsymbol{A} \boldsymbol{b}, \boldsymbol{B} \boldsymbol{b}, \boldsymbol{C} \boldsymbol{b}$, Detail profiles of the subfields with the (Figure legend continues.) 
A

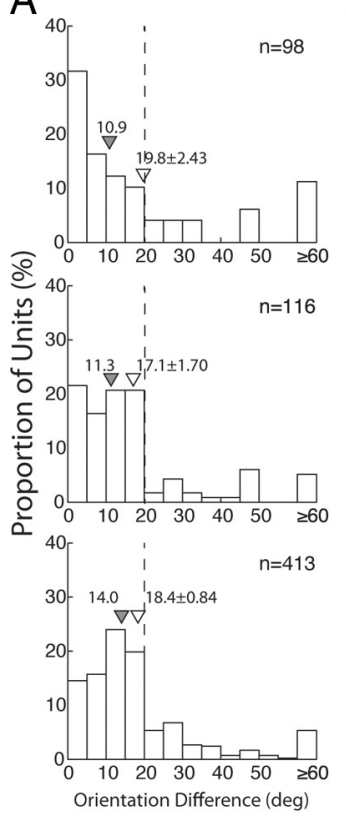

B

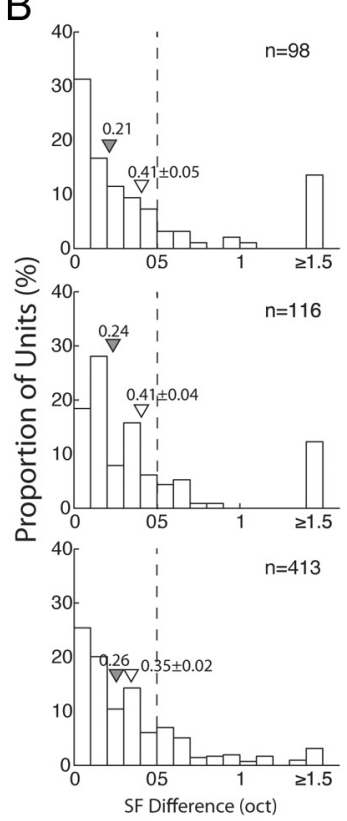

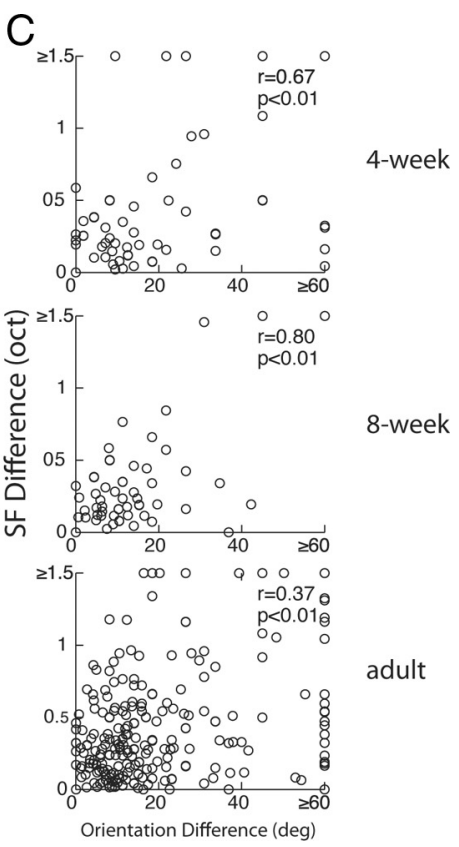

Figure 4. Spatial homogeneity of local spectral selectivity maps with facilitatory profiles across the receptive fields in infants and adults. $\boldsymbol{A}$, Histogram illustrating the distribution of the maximum orientation differences between neighboring pairs of subfields. $\boldsymbol{B}$, Distribution of the maximum spatial frequency differences between neighboring pairs of subfields. $\boldsymbol{C}$, The maximum orientation differences between a pair of neighboring subfields within each neuron are on the $x$-axis, and SF differences are on the $y$-axis. Filled triangles indicate median values, and open triangles indicate means ( \pm SE). Note that several units in each age group contained just one subfield, hence lowering the total number of units for this analysis.

To evaluate the significance of the spike-triggered signals, we calculated the average and SD (noise level) of signals using shuffled correlations. We obtained the shuffled correlations by calculating cross-correlations between spike trains and shifted (unpaired) stimulus blocks. The mean and SD of the shuffled correlations were then used to normalize the original spike-triggered signals into $z$-score representations. To reduce the computational burden, we assumed that the noise level was identical for a sequence of random patterns for any given subfield and spatial frequency.

The $z$-scores were used to represent the response strength in the spectral receptive field profiles, taking variability and statistical significance of responses into account. The $z$-scores were sometimes negative, which was interpreted as a reduction of activities below the baseline level. The statistical significance of signals was examined by the $z$-score, corrected for multiple comparisons by Bonferroni's method (Fig. $1 B$, right). The degree of freedom for the Bonferroni's correction was set to the number of subfields multiplied by the number of noise elements within \pm 1 SD of the analyzing Gaussian window. Black lines in the LSRC plot indicate contours for $p=0.05$.

\section{Anatomical methods}

To identify recording sites, small electrolytic lesions were produced at three locations along the electrode track (at the end of each penetration, at the mid-point of the penetration, and near the cortical surface) by passing current through the electrode $(5 \mu \mathrm{A}$ for $<5 \mathrm{~s}$, electrode tip negative). At the end of the recording experiments, an overdose of sodium pentobarbital $(100 \mathrm{mg} / \mathrm{kg})$ was administered intravenously to induce a

(Figure legend continued.) maximum $z$-score. $A c, B C, C c$, Schematic diagrams showing the preferred orientations (bar angle) and spatial frequencies (width), and the maximum $z$-scores (saturation) of the subfields. $\boldsymbol{D a}, \boldsymbol{D b}$, Schematic diagrams showing the preferred orientation (bar angle) and spatial frequencies (width), and the maximum $z$-scores (saturation) of the subfields in a V2 neuron from an 8-week-old infant with highly homogeneous subfield matrix $(\boldsymbol{D a})$ and a neuron with a heterogeneous subfield matrix (Db). c/d, Cycles per degree; deg, degree. deep level of anesthesia and the animals were killed. The animals were perfused through the heart with an aldehyde fixative ( $2 \%$ paraformaldehyde and $0.5 \%$ glutaraldehyde in $0.1 \mathrm{M}$ phosphate buffer, $\mathrm{pH}$ 7.4). The brains were removed immediately and kept overnight in fixative with $20 \%$ sucrose. The tissues were cut in $40 \mu \mathrm{m}$ sections on a freezing microtome in parasagittal planes.

\section{Results}

We analyzed the spatial matrix of subfields for 110 V2 neurons from 4-weekold monkeys and 119 neurons from 8-week-old monkeys. The data from infants were compared with those obtained in adult monkeys (416 neurons). It should be noted that about one-third of the data from adult monkeys came from our previous study, and that the results for adult monkeys in the current study were very similar to those previously published (Tao et al., 2012).

\section{Local spectral selectivity maps with facilitatory profiles}

The spatial matrices of facilitatory subfields of V2 neurons were largely adultlike as early as 4 weeks of age. Figures 2 and 3 illustrate the similarities and differences in spatial organization of subfields between infants and adults. The spatial matrix of facilitatory subfields of a typical adult V2 neuron shows a high degree of homogeneity, as indicated by similar local preferences to orientation at different positions across the receptive field (Fig. 2A). The subfield located near the center of the matrix showed the highest levels of activation ( $z$-max), and the $z$-score decreased toward the periphery of the subfield map (Fig. $2 A a-A c$ ). The largest orientation difference between the neighboring subfields for this representative was $11.3^{\circ}$, while the largest optimal spatial frequency difference was 0.77 octaves (Fig. 2Ac). In contrast, another V2 neuron from an adult monkey exhibited a more complex spatial matrix of facilitatory subfields (Fig. $2 \mathrm{~B}$ ). The spatial matrix of this unit consisted of several subfields that are nearly orthogonal to each other in their preferred orientations (Fig. $2 B a, B c$ ). These neurons could potentially show higher sensitivities to local line elements embedded in small regions of complex stimuli that substantially differ in orientation (Ito and Komatsu, 2004; Anzai et al., 2007; El-Shamayleh et al., 2011; Tao et al., 2012).

At 4 weeks of age, the spatial matrix of facilitatory subfields in the majority of V2 neurons was similar to that in adults. However, the strength of responses ( $z$-max) was lower compared with adults. The spatial matrix of facilitatory subfields of a typical neuron from a 4-week-old infant was quite homogeneous. For the representative neuron shown in Figure $3 A$, the largest orientation and optimal spatial frequency differences between adjacent subfields were $0^{\circ}$ and 0.41 octaves, respectively (Fig. $3 A a, A c$ ). However, the response strength of the subfield with the highest $z$-score was weaker than that typically found in adults (Fig. $3 A b$ ). As in adults, we found a subset of neurons exhibiting relatively large orientation and spatial frequency differences between neighboring subfields. For the example neuron illustrated in Figure $3 B$, the largest orientation difference was $33.7^{\circ}$ and the largest 


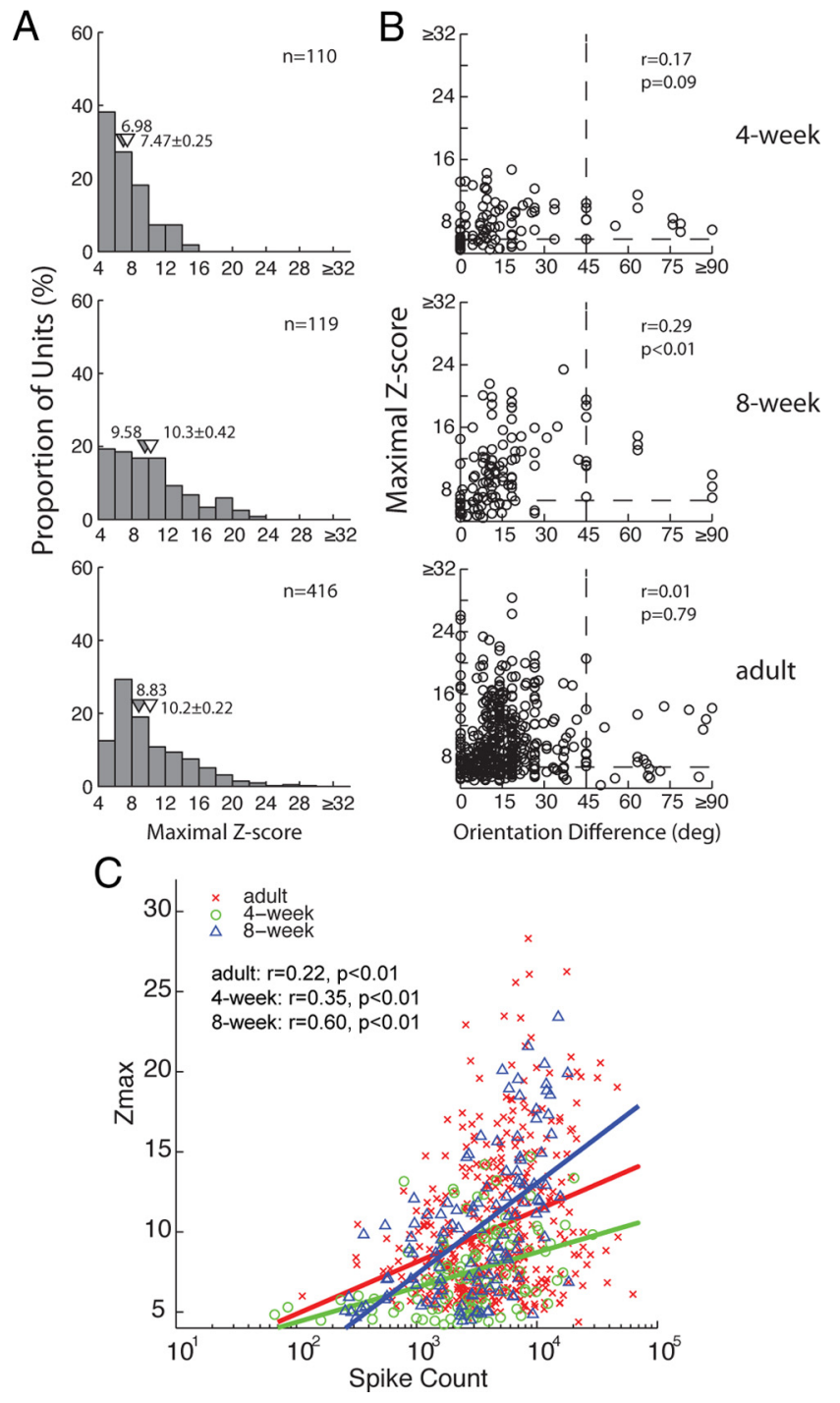

Figure 5. Comparisons of response strength and reliability (z-max) of V2 neurons in infants and adults. $\boldsymbol{A}$, Histograms illustrating the distribution of $z$-max scores in adults (bottom), 4-week-old (top) and 8-week-old infants (middle). $\boldsymbol{B}$, Scatter plots relating z-max scores of individual neurons with their maximum orientation differences between neighboring subfields. C, The z-max scores of individual neurons as a function of their total spike counts.

optimal spatial frequency difference was 0.58 octaves (Fig. $3 B a, B c$ ). Also note a gradual "shift" in the preferred orientations of the four subfields in the right position $(x$ position $=1.3)$ of the matrix (Fig. 3Bc). Another example of a spatial matrix of subfields for a neuron from a 4-week-old infant had only four subfields and a relatively low $z$-max score (Fig. $3 \mathrm{Ca}, \mathrm{Cb}$ ). Interestingly, the orientation differences between these subfields were as large as $76.0^{\circ}$ (Fig. $3 C c$ ).

In 8-week-old infants, the homogeneity of the subfield matrices was indistinguishable from that in adults; the majority of units showed high homogeneity, and a subset of V2 neurons had relatively large orientation and spatial frequency differences between neighboring subfields. For the example neuron in Figure $3 \mathrm{Da}$, the largest orientation and optimal spatial frequency differences between adjacent subfields were $0^{\circ}$ and 0 octaves. And as illustrated by the unit in Figure $3 \mathrm{Db}$, some units exhibited relatively heterogeneous subfield matrices. The largest orientation and optimal spatial frequency difference for this neuron were $63.4^{\circ}$ and 1.96 octaves, respectively. However, the strength of responses was in general not different from that in adults (see Fig. 5; rank-sum test, $p>0.96$ ).

\section{Differences in preferred orientation and spatial frequency}

For our population analysis, the local variations in preferred orientation and spatial frequency between subfields were analyzed for the different age groups by calculating the largest differences between any pair of neighboring subfields in a matrix (Fig. 4). About 25\% of V2 neurons in 4- and 8-week-old infants exhibited substantial differences in preferred orientation and spatial frequency between a pair of facilitatory subfields. However, as in adults, the majority of V2 neurons in all infants exhibited relatively homogeneous matrices of facilitatory subfields; that is, the largest differences in preferred orientation (Fig. 4A) and spatial frequency (Fig. $4 B$ ) were relatively small. Most importantly, the frequency distributions of the orientation and spatial frequency differences in 4- and 8-week-old monkeys were not significantly different from that in adults (rank-sum test, $p>0.06$ ). A neuron with a large maximum orientation difference tended to have a large maximum spatial frequency difference (Fig. 4C). Therefore, for our populations of V2 neurons, there was a significant positive relationship between these two values, and interestingly, the correlations were relatively better in infants than in adults.

\section{The strength and variability of responses}

At 4 weeks of age, the responsiveness of V2 neurons measured with high-contrast drifting or stationary gratings is relatively poor (Zhang et al., 2005, 2008; Maruko et al., 2008). To determine how these lower firing rates could affect the homogeneity of the V2 subfield matrix, we quantitatively analyzed the $z$-scores for each neuron. The median $z$-max score 4 -week-old infants was significantly lower than that for 8-week-old infants or for adults (rank-sum test, $p<0.0001$ ) (Fig. 5A). Moreover, we did not find any neurons with $z$-max score higher than 16 in 4-week-old monkeys. By 8 weeks of age, the distribution of $z$-max values was similar to that in adults (rank-sum test, $p>0.96$ ).

The scatter plots of the $z$-max scores as a function of the maximum orientation difference between neighboring subfields shows that there was little or no significant correlation between the two values in infants or adults (Fig. $5 B$ ). In 4 -week-old infant monkeys, neurons with large orientation differences between adjacent subfields (e.g., $\geq 45^{\circ}$ ) had $z$-max scores similar to those units with smaller orientation differences. Therefore, the strength of activation ( $z$-max) did not have a consistent relationship with the homogeneity of the subfield matrices. Finally, the $z$-max value of a unit was broadly correlated with the total spike count in both infants and adults, but the observed relationship was not systematically related to the monkeys' ages (Fig. 5C).

\section{Local spectral selectivity maps with suppressive profiles}

The majority of V2 neurons in both 4- and 8-week-old infants had subfields with suppressive profiles in their local spectral sensitivity maps (subfields). A typical spatial matrix with suppressive (blue) and facilitatory (red) subfield profiles for a representative neuron from a 4-week-old infant is illustrated in Figure 6. Our cell population analysis shows that the percentage of V2 neurons having suppressive subfield profiles was significantly less (20\%) in 4-week-old infant monkeys than in adults (40\%) if the measurement was made at the optimal correlation delay for facilitatory profiles ( $\chi^{2}$ test, $p<0.001$ ) (Fig. $7 A$; for a further analysis of this issue, also see Fig. 9). At 8 weeks of age, the proportion increased to $30 \%$, which was not significantly different from that in adults $\left(\chi^{2}\right.$ test, $\left.p>0.2\right)$. The difference between 4 -week-old 
A

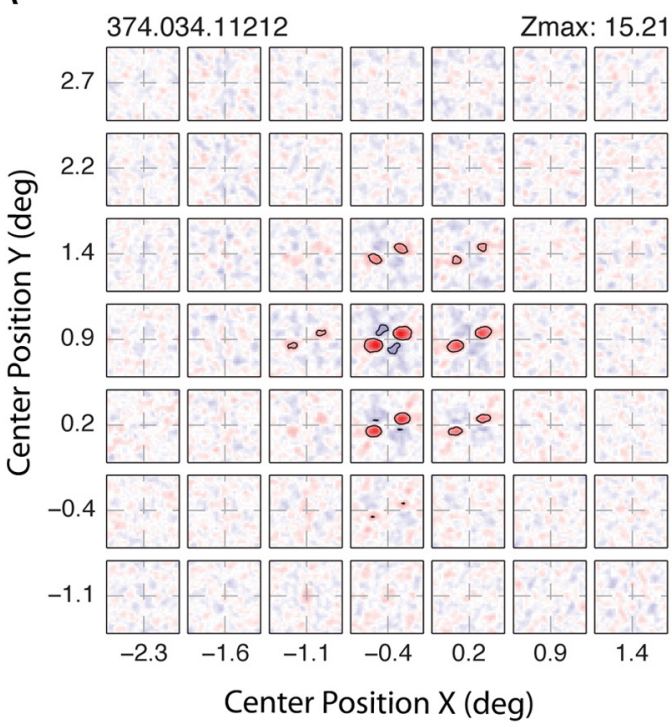

B Fac. OR: $112(\mathrm{deg})$ SF: $0.93(\mathrm{c} / \mathrm{d})$

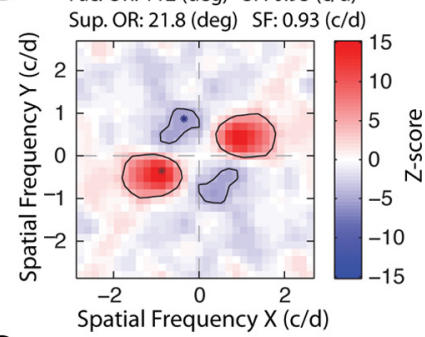

C

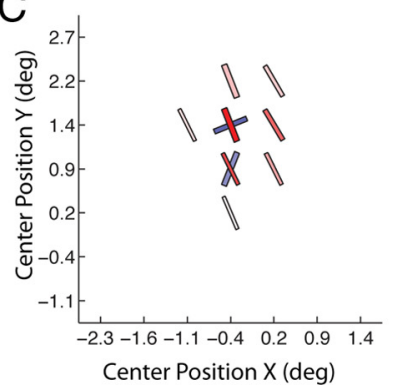

Figure 6. A V2 neuron from a 4-week-old infant having subfields with both facilitatory and suppressive profiles. $A$, Spatial matrix of subfields with both profiles. $\boldsymbol{B}$, Detailed profile of the subfield with the maximum $z$-scores. Location of the highest and lowest z-scores is indicated with asterisks. C, Schematic diagram of the preferred orientations (bar angles) and spatial frequencies (widths) of subfields with the faicilitatory (red) and suppressive (blue) profiles.
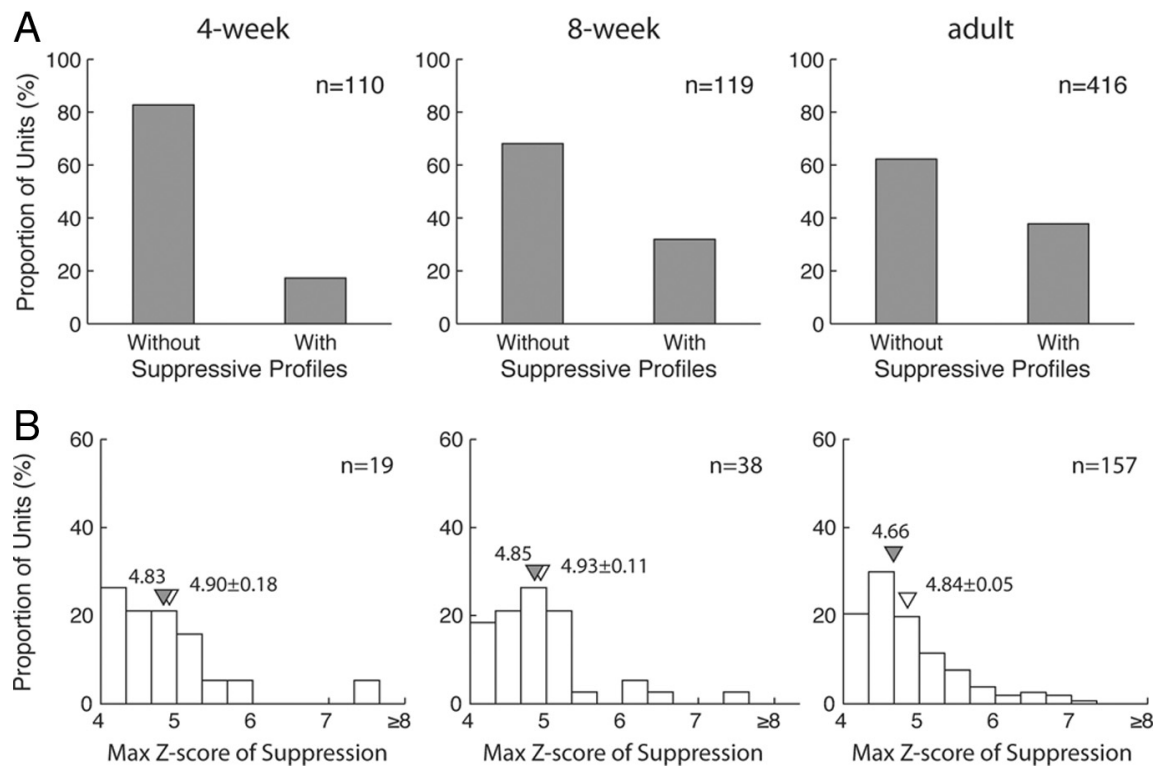

Figure 7. Development of suppressive subfield profiles. $A$, Proportion of $V 2$ neurons having subfields with facilitatory profiles alone (left) or with both facilitatory and suppressive profiles (right) in infants and adults. $\boldsymbol{B}$, Comparisons of $z$-max scores of suppressive subfield profiles. Filled triangles indicate median values and open triangles indicate means ( $\pm \mathrm{SE}$ ).

and 8-week-old monkeys was also significant $\left(\chi^{2}\right.$ test, $\left.p<0.02\right)$. Although the percentage of V2 neurons with suppressive subfields was significantly less in 4-week-old infant monkeys, the overall (average or median) strength of suppressive profiles $(z$-max $)$ at 4 weeks of age was not different from that in adults or 8 -week-old infants (rank-sum test, $p>0.3$ ) (Fig. $7 B$ ).

\section{Relationships between facilitatory and suppressive subfield profiles}

Tuned suppression in the receptive fields of V2 neurons is thought to be involved in the emergence of stimulus selectivity for complex stimulus features (Anzai et al., 2007; Willmore et al.,
2010; Tao et al., 2012). To gain insights into the role of suppression in the processing of complex stimulus features during early development, we analyzed the relationship between the facilitatory and suppressive subfields in individual V2 receptive-fields. We paid special attention to (1) the spatial relationships between the facilitatory and suppressive profiles within a spectral map (orientation and spatial frequency differences); (2) the differences in the strength of activation ( $z$-max values); and (3) the dynamics (timing differences) of the peak facilitatory and suppressive responses.

\section{Differences in orientation, spatial frequency, and activation strength} One of the more significant findings on the relationships between suppressive and facilitatory profiles of subfields was that in adults nearly $60 \%$ of sampled neurons showed the maximal orientation differences between the facilitatory and suppressive profiles that were $>60^{\circ}$ (Figs. 6, $8 A)$. This relationship resembles classical cross-orientation suppression. In 4- and 8 -week-old infants, there were similar relationships between facilitation and suppression, and the percentage of neurons with the orientation difference $>80^{\circ}$ was even higher $(27 \%)$ than in adults $(17 \%)$. More importantly, the distribution of orientation differences between facilitation and suppression had a continuous and broad range, from $<10^{\circ}$ to $90^{\circ}$. It is also important to note that there was no statistically significant difference in this distribution between any age group (rank-sum test, $p>0.2$ ). The observed heterogeneous arrays of suppressive profiles could alter the preferred orientations of spiking output of facilitatory subfields, hence resulting in a spatial matrix for a given neuron that exhibits higher sensitivity to complex stimulus features.

The preferred spatial frequencies for suppressive profiles were substantially higher than those for facilitatory profiles in the great majority of subfields ( $t$ tests: $p<0.0001$ for adults, $p<0.004$ for 4 -week-old, $p<0.0001$ for 8 -week-old) (Figs. 6, $8 B$ ). This result is consistent with a previous study in adult V2 (Willmore et al., 2010). Figure $8 B$ also shows that these spatial frequency differences between facilitatory and suppressive profiles were very similar in all age groups.

In adult V2, the relative strength of responses $(z$-max) for suppressive profiles varied considerably between neurons, but on the average, it was about one-half of the response strength of facilitatory profiles (Fig. 8C). Moreover, the median or mean ratios of $z$-max for suppression over $z$-max for facilitation in the infant monkeys were not substantially different from the compa- 
A
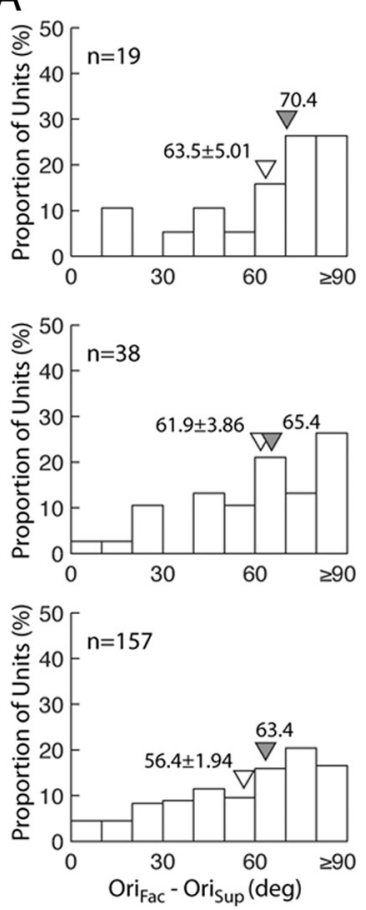

$\mathrm{B}$
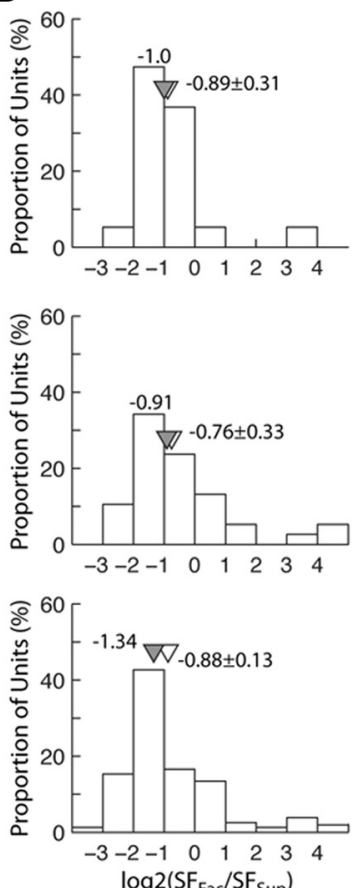

C
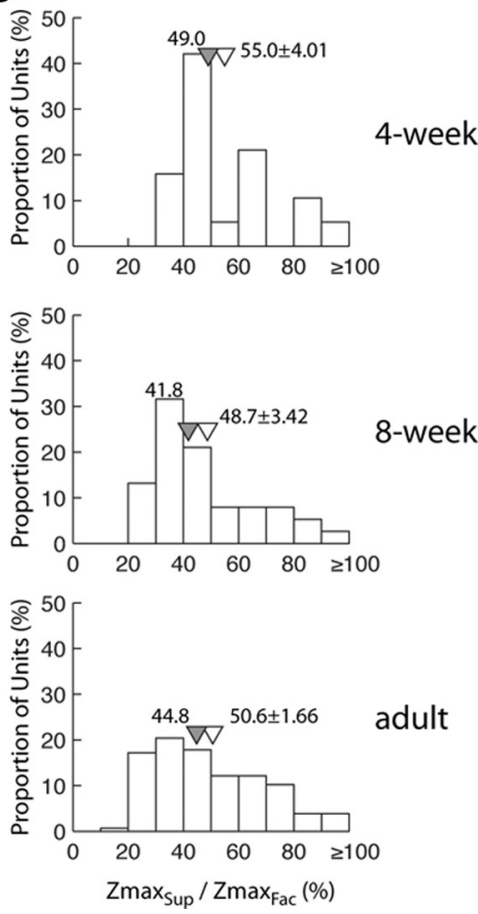

Figure 8. Relationships between the suppressive profiles and the facilitatory profiles of subfields for individual V2 neurons in infants and adults. $\boldsymbol{A}$, Distribution of differences in the preferred orientation of subfields between facilitatory and suppressive profiles. $\boldsymbol{B}$, Distributions of the preferred spatial frequency differences of subfields between facilitatory and suppressive profiles. $\boldsymbol{C}$, Distribution of the ratios of maximum suppressive $z$-scores over maximum facilitatory $z$-scores. Filled triangles indicate median values, and open triangles indicate means ( \pm SE).

rable values in adults (rank-sum test, $p>$ 0.2 ; $t$ test, $p>0.3$ ). These results suggest that the key relationships between facilitatory and suppressive profiles in V2 neuron subfields are largely adult-like as early as 4 weeks of age.

\section{Dynamics of peak facilitatory and suppressive responses}

The relative timing ("dynamics") of facilitation and suppression was analyzed by comparing the peak correlation delays for these responses (Fig. 9). To quantify the correlation delays, we measured the $z$-max values for the facilitatory and suppressive profiles at all correlation delays between 30 and $150 \mathrm{~ms}$ in $15 \mathrm{~ms}$ steps. Then we determined the correlation delay ("latency") at which the $z$-score was highest for facilitatory and suppressive profiles. Figure $9 A$ illustrates examples of the three different timing relations in 4-week-old infants; suppression peaked before facilitation (left), the optimal correlation delay was the same for suppression and facilitation (middle), and there was a substantially longer delay for the suppressive profiles (right). Neurons with these patterns of peak correlation delays were found in all infants and adults. Because the correlation delays were calculated in $15 \mathrm{~ms}$ steps, it is important to keep in mind that the real timing difference could be off by $\pm 7.5 \mathrm{~ms}$ (see Materials and Methods). In light of these
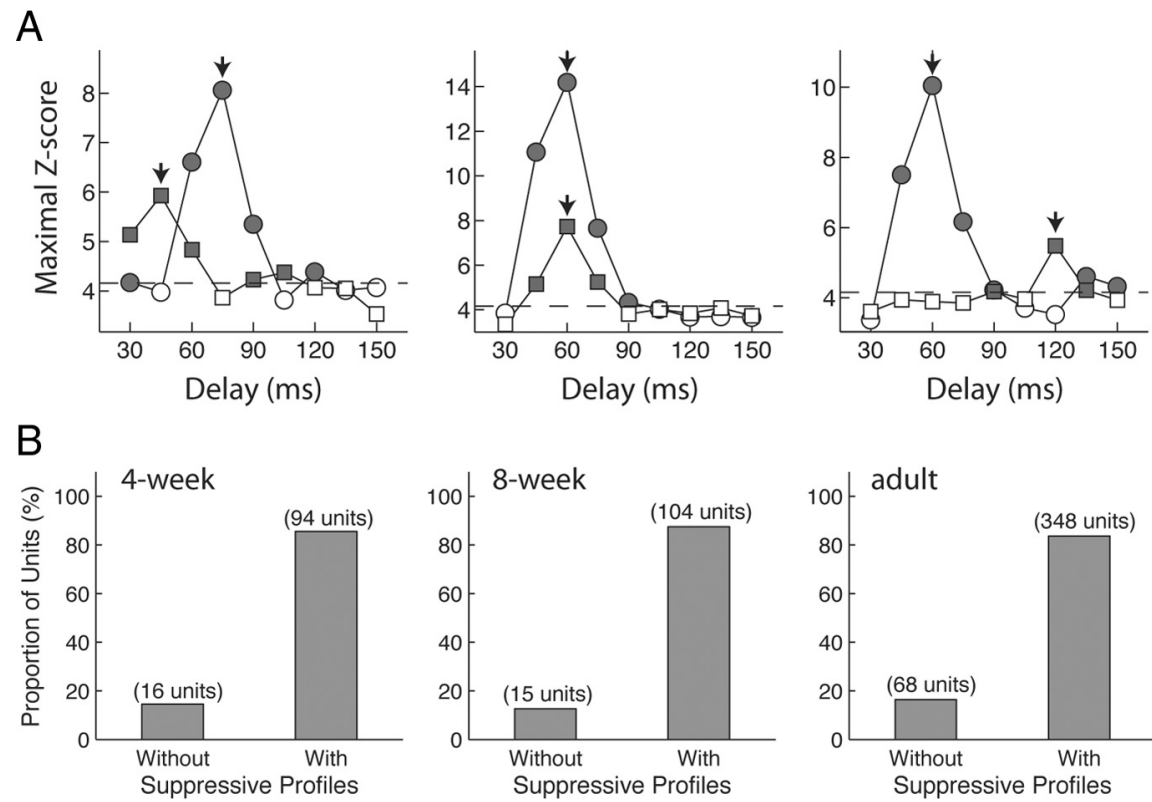

Figure 9. Differences in correlation delays (latency) between the facilitatory and suppressive subfields in infants and adults. $\boldsymbol{A}$, Examples of correlation delays. The z-max values at different delays for facilitatory (circles) and suppressive (squares) profiles. Filled data points signify z-max values that are significant. Arrows indicate the latency at which the peak response occurred for facilitatory and suppressive profile, respectively. $\boldsymbol{B}$, The proportion of $\mathrm{V} 2$ neurons with and without suppressive subfield profiles in 4-week-old (left), 8-week-old infants (middle), and adults (right).
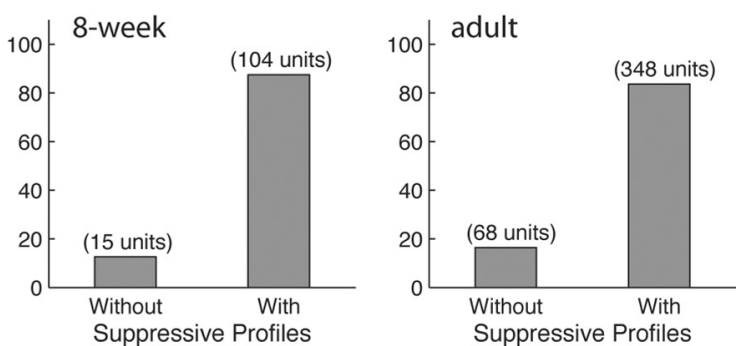

different correlation delays between facilitation and suppression, we re-examined the proportion of V2 neurons with suppressive profiles by taking into consideration the timing of the peak response for the suppressive profile relative to that for the facilitatory profile (Fig. 9B). The results are strikingly different from the analysis shown in Figure $7 A$ where the proportion of units with suppressive 
A
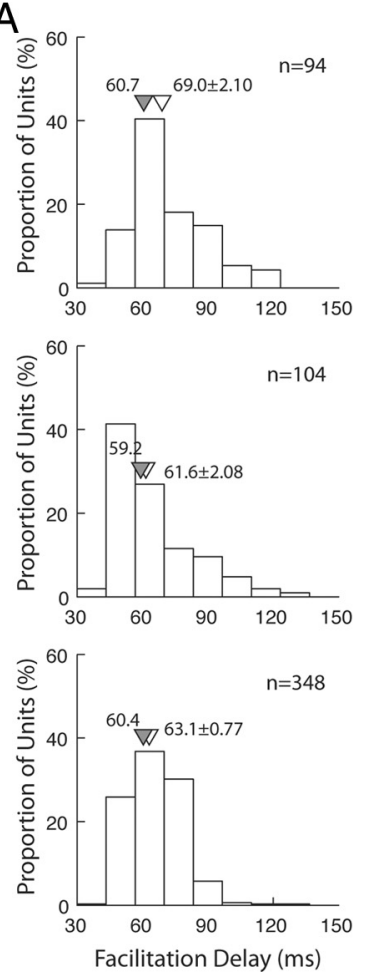

$\mathrm{B}$
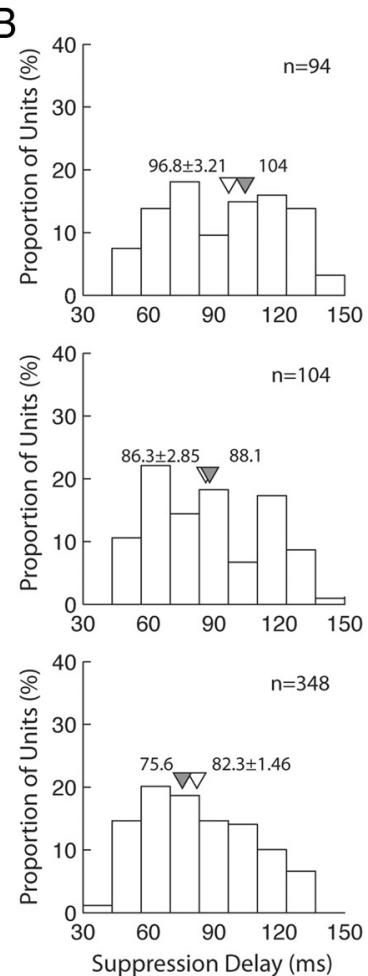

C
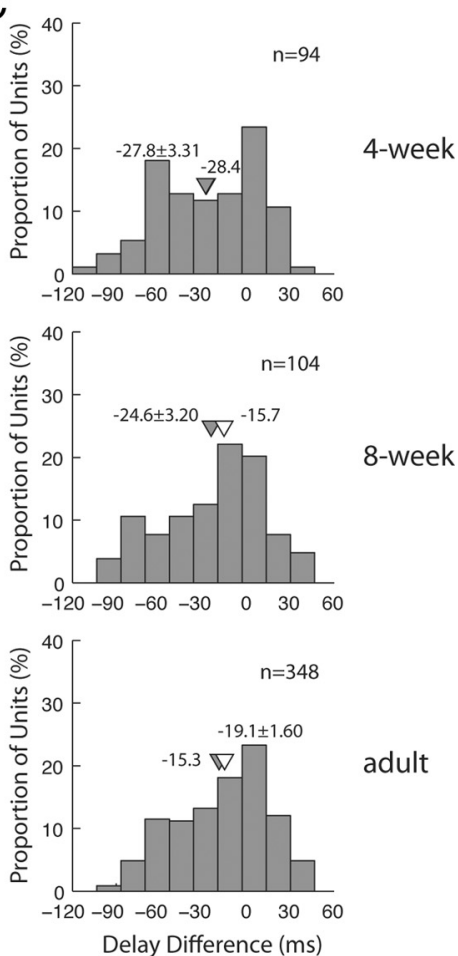

Figure 10. Differences in correlation delays (latency) between the facilitatory and suppressive subfields in infants and adults. $\boldsymbol{A}$, Distribution of the correlation delays (latency) for facilitatory subfields in infants and adults. $\boldsymbol{B}$, Distribution of the correlation delays (latency) for suppressive subfields in infants and adults. $\boldsymbol{C}$, Differences in correlation delays between facilitatory and suppressive profiles in infants and adults. Triangle signifies median value, and circle signifies the mean. Filled triangles indicate median values, and open triangles indicate means ( \pm SE).

profiles was determined at the peak correlation delay for the facilitatory subfield profiles.

About $80 \%$ of V2 neurons in both adults and infants had spatial matrices containing suppressive profiles, but the latency for maximum suppression was longer in infants relative to that for facilitation. In Figure 10, we show the results of our population analysis on the development of the timing of the optimal correlation delays for facilitatory profiles (Fig. 10A), for suppressive profiles (Fig. 10B), and the differences between these two profiles (Fig. 10C). For facilitatory profiles, the mean ( $\pm \mathrm{SE}$ ) correlation delay in 4-week-old monkeys was slightly, but significantly, longer than that in adults ( $t$ test, $p=0.0015)$. However, the mean $( \pm \mathrm{SE})$ correlation delay for suppressive profiles was much longer in 4-week-old-infants than that in adults $(t$ test, $p<$ $0.0001)$. Consequently, the differences in optimal correlation delays between the facilitatory and suppressive profiles (facilitatory - suppressive) in 4-week-old infants were significantly greater than that in adults $(t$ test, $p<0.014)$. There was no difference between 8 -week-old infants and adults in any of these comparisons ( $t$ test, $p>0.1$ ).

\section{Discussion}

The most important finding of this study was that the spatial organization of the subfields within the RFs of V2 neurons is largely adult-like as early as 4 weeks of age. While the majority of V2 neurons in all infants had relatively homogeneous matrices of facilitatory subfields, a similar proportion of V2 neurons in infants and adults $(20-30 \%)$ showed relatively large differences in the preferred orientation and the optimal spatial frequency between neighboring subfields. The results in adults complement previous work in adult monkeys using "conventional" stimuli, which demonstrated that about one-third of V2 neurons respond better to a combination of luminance elements having substantial orientation differences (Ito and Komatsu, 2004; Anzai et al., 2007; Hegdé and Van Essen, 2007). Here we showed that this sort of "unique" sensitivity to angled contour elements is likely to exist in V2 neurons as early as 4 weeks of age, although we did not directly measure neuronal responses using "angled" contour stimuli.

We also found subtle immaturities in certain neuronal responses in infant monkeys. One of the more subtle immaturities in infant V2 was that $z$-max scores for facilitatory subfields in 4 -week-old-monkeys were significantly lower than those in adults. The $z$-max scores were used to represent the response strengths of the subfield profiles, taking variability and statistical significance of responses into consideration (Nishimoto et al., 2006; Tao et al., 2012). This finding is consistent with our previous observations on V2 neurons in infants where we found higher contrast thresholds for RF centers and lower sustained discharge rate in response to stationary or drifting sine wave gratings (Zhang et al., 2005, 2008; Maruko et al., 2008). However, the subnormal responsiveness of V2 neurons in 4-week-old infants did not influence the overall homogeneity of the subfield matrix (Fig. 5B). This is not inconsistent with some of the previous observations for cortical neurons of infant monkeys in that while the firing rates of V1 and V2 neurons in 1- and 4-week-old monkeys are much lower than in adults, the lower response rate increases the reliability of responses; fewer action potentials are required to "convey" the same amount of information (Rust et al., 2002; Maruko et al., 2008).

Another remarkable result was that the differences in preferred orientation between facilitatory and suppressive subfield profiles were often relatively large (e.g., $>60^{\circ}$ ), and these differ- 
ences were virtually the same for infants and adults (Fig. 8). Equally important, the orientation differences between facilitation and suppression extended over a broad range in both infants and adults $(\sim 30-40 \%$ of $\mathrm{V} 2$ neurons showed the orientation differences that were distributed between $10^{\circ}$ and $60^{\circ}$ ). This broad range of heterogeneous arrays of suppressive profiles in a V2 neuron could interact with facilitatory profiles that show high homogeneity. As a result, the optimal orientation for the spiking output signals from these homogeneous facilitatory subfields are altered in a complex manner, and the entire spatial matrix becomes more heterogeneous. These neurons with "tuned" suppression are likely to become more sensitive to a wide range of corners and angles embedded in complex stimuli both in infants and adults (Willmore et al., 2010).

In adults, the relative strength of suppressive profiles was, on average, about one-half of that for facilitatory profiles, possibly reflecting a limit imposed by extracellular recording methods. However, this relationship was basically unchanged during the development (Fig. 8). Since the $z$-max values of facilitatory profiles were significantly lower at 4 weeks of age than in adults, the $z$-max values for suppressive profiles were also proportionally lower in each neuron. These results suggest that the observed relationships between suppressive and facilitatory profiles were established in V2 neurons relatively early after birth and do not substantially change during early development.

The interpretation of the interactions between suppressive and facilitatory profiles of subfields that are revealed by the LSRC method must take into account critical methodological limitations. In particular, the LSRC method calculates the net sum of facilitation and suppression for each frequency, and, therefore, it can only visualize whichever is stronger (Nishimoto et al., 2006; Tao et al., 2012). However, it is important to keep in mind that the summed information reflects the spiking output of neurons that is transmitted to the next neurons in the cascade of cortical processing. Therefore, the information that neurons in a higherorder visual area (e.g., V4, MT) receive from V2 neurons in both infants and adults is that the orientation and spatial frequency differences between suppressive and facilitatory profiles of subfields cover a broad range and are very large in the majority of V2 neurons.

The average peak correlation delays for facilitatory subfields at 4 weeks of age were slightly but significantly longer than in adults. This is generally consistent with our previous finding that the average onset and peak response latencies of V2 neurons to drifting high-contrast gratings in 4-week-old monkeys were significantly longer than in adults (Zheng et al., 2007; Zhang et al., 2008). Also the average peak correlation delays for suppressive subfield profiles at 4 weeks of age were even longer than in adults. Consequently, the differences in the correlation delays between 4-week-old infants and adults were much greater for suppressive subfields than that for facilitatory subfields (Fig. 10). The neural basis of the relatively longer latency for suppression is unclear, primarily because we do not know the cortical connections generating the suppressive subfield profiles of V2 neurons. The possible connections include the feedforward inhibition that is part of converging V1 inputs, local and long-range inhibitory networks within V2, and feedback connections from higher-order visual areas. These are not mutually exclusive and likely to combine with different weights, as shown in adult V1 (Malone and Ringach, 2008). The overall longer delays in both facilitatory and suppressive subfields in 4-week-old infants could reflect the less refined projection fibers from V1 (Baldwin et al., 2012). However, this explanation does not apply to the longer delays for suppressive subfields relative to that for facilitatory subfields because the correlation delays should be similar for facilitation and suppression. Immaturities in the feedback connections from higher-order visual areas can contribute to the enhanced delay in suppression because the overall maturation of higher-order visual areas downstream from V2 is slower (Barone et al., 1996; Batardière et al., 2002; Kiorpes and Movshon, 2003; Zhang et al., 2005; Zheng et al., 2007). Also, immaturities in the local and long-range connections within V2 could be a source of longer delays in suppression relative to facilitatory subfields (WongRiley, 1979; Matsubara et al., 1985; Rockland, 1985; Hubel and Livingstone, 1987; Amir et al., 1993; Malach et al., 1994; Baldwin et al., 2012). However, the functional significance of these connections has not been extensively explored in adult V2.

\section{Perceptual implications}

As mentioned earlier, a substantial proportion of V2 neurons in adult monkeys is sensitive to corners or angled contour elements that make up a part of global forms (Ito and Komatsu, 2004; Anzai et al., 2007; Willmore et al., 2010; El-Shamayleh et al., 2011). These neurons are considered to play a key role in processing local information necessary for detecting and/or discriminating texture-defined form patterns, and infant monkeys are capable of discriminating texture-defined visual borders as early as 6 weeks of age (El-Shamayleh et al., 2010). Similar observations were made in human infants; infants can detect texture-defined patterns as early as 3-5 months of age (approximately equivalent to 3-5 weeks of age in monkeys) (Norcia et al., 2005; Sireteanu et al., 2005). The results in this study parallel these psychophysical observations. While moderate immaturities are present, V2 neurons in 4-week-old monkeys have RF structures that are basically similar to those in adults. These observations suggest that V2 neurons in 4-week-old monkeys are likely to be sensitive to angled neighboring contour elements. If "simple mechanisms that can compare the local content of neighboring image sub-regions" are required for the initial processing of complex features for texture perception (El-Shamayleh et al., 2011), such mechanisms exist in primate $\mathrm{V} 2$ as early as 4 weeks of age.

\section{References}

Amir Y, Harel M, Malach R (1993) Cortical hierarchy reflected in the organization of intrinsic connections in macaque monkey visual-cortex. J Comp Neurol 334:19-46. CrossRef Medline

Anzai A, Peng X, Van Essen DC (2007) Neurons in monkey visual area V2 encode combinations of orientations. Nat Neurosci 10:1313-1321. CrossRef Medline

Atkinson J, Hood B, Wattam-Bell J, Anker S, Tricklebank J (1988) Development of orientation discrimination in infancy. Perception 17:587-595. CrossRef Medline

Baldwin MK, Kaskan PM, Zhang B, Chino YM, Kaas JH (2012) Cortical and subcortical connections of V1 and V2 in early postnatal macaque monkeys. J Comp Neurol 520:544-569. CrossRef Medline

Barone P, Dehay C, Berland M, Kennedy H (1996) Role of directed growth and target selection in the formation of cortical pathways: prenatal development of the projection of area V2 to area V4 in the monkey. J Comp Neurol 374:1-20. CrossRef Medline

Batardière A, Barone P, Knoblauch K, Giroud P, Berland M, Dumas AM, Kennedy H (2002) Early specification of the hierarchical organization of visual cortical areas in the macaque monkey. Cereb Cortex 12:453-465. CrossRef Medline

DeAngelis GC, Ohzawa I, Freeman RD (1993) Spatiotemporal organization of simple-cell receptive fields in the cat's striate cortex. I. General characteristics and postnatal development. J Neurophysiol 69:1091-1117. Medline

El-Shamayleh Y, Movshon JA (2011) Neuronal responses to texturedefined form in macaque visual area V2. J Neurosci 31:8543-8555. CrossRef Medline 
El-Shamayleh Y, Movshon JA, Kiorpes L (2010) Development of sensitivity to visual texture modulation in macaque monkeys. J Vis 10(11):11 11. CrossRef Medline

Gallant JL, Braun J, Van Essen DC (1993) Selectivity for polar, hyperbolic, and Cartesian gratings in macaque visual cortex. Science 259:100-103. CrossRef Medline

Gallant JL, Connor CE, Rakshit S, Lewis JW, Van Essen DC (1996) Neural response to polar, hyperbolic, and Cartesian gratings in are $\mathrm{V} 4$ of macaque monkey. J Neurophysiol 76:2718-2739. Medline

Geisler WS (2008) Visual perception and the statistical properties of natural scenes. Annu Rev Psychol 59:167-192. CrossRef Medline

Geisler WS, Perry JS, Super BJ, Gallogly DP (2001) Edge co-occurrence in natural images predicts contour grouping performance. Vision Res 41: 711-724. CrossRef Medline

Hegdé J, Van Essen DC (2007) A comparative study of shape representation in macaque visual areas v2 and v4. Cereb Cortex 17:1100-1116. CrossRef Medline

Hou C, Pettet MW, Sampath V, Candy TR, Norcia AM (2003) Development of the spatial organization and dynamics of lateral interactions in the human visual system. J Neurosci 23:8630-8640. Medline

Hubel DH, Livingstone MS (1987) Segregation of form, color, and stereopsis in primate area-18. J Neurosci 7:3378-3415. Medline

Ing AD, Wilson JA, Geisler WS (2010) Region grouping in natural foliage scenes: image statistics and human performance. J Vis 10(4):10 1-19. CrossRef Medline

Ito M, Komatsu H (2004) Representation of angles embedded within contour stimuli in area V2 of macaque monkeys. J Neurosci 24:3313-3324. CrossRef Medline

Kiorpes L, Movshon JA (2003) Neural limitations on visual development in primates. In: The visual neurosciences (Chalupa LM, Werner JS, eds), pp 159-173. Cambridge, MA: MIT Press.

Malach R, Tootell RB, Malonek D (1994) Relationship between orientation domains, cytochrome-oxidase stripes, and intrinsic horizontal connections in squirrel-monkey area V2. Cereb Cortex 4:151-165. CrossRef Medline

Malone BJ, Ringach DL (2008) Dynamics of tuning in the Fourier domain. J Neurophysiol 100:239-248. CrossRef Medline

Maruko I, Zhang B, Tao X, Tong J, Smith EL 3rd, Chino YM (2008) Postnatal development of disparity sensitivity in visual area 2 (v2) of macaque monkeys. J Neurophysiol 100:2486-2495. CrossRef Medline

Matsubara J, Cynader M, Swindale NV, Stryker MP (1985) Intrinsic projections within visual-cortex-evidence for orientation-specific local connections. Proc Natl Acad Sci U S A 82:935-939. CrossRef Medline
Nishimoto S, Ishida T, Ohzawa I (2006) Receptive field properties of neurons in the early visual cortex revealed by local spectral reverse correlation. J Neurosci 26:3269-3280. CrossRef Medline

Norcia AM, Pei F, Bonneh Y, Hou C, Sampath V, Pettet MW (2005) Development of sensitivity to texture and contour information in the human infant. J Cogn Neurosci 17:569-579. CrossRef Medline

Pasupathy A, Connor CE (2001) Shape representation in area V4: positionspecific tuning for boundary conformation. J Neurophysiol 86:25052519. Medline

Pasupathy A, Connor CE (2002) Population coding of shape in area V4. Nat Neurosci 5:1332-1338. CrossRef Medline

Press WH, Flannery BP, Teukolsky SA, Vetterling WT (1992) Fast Fourier transform. In: Numerical recipes in Fortran: the art of scientific computing, pp 490-529. Cambridge, UK: Cambridge UP.

Rockland KS (1985) A reticular pattern of intrinsic connections in primate area-V2 (area-18). J Comp Neurol 235:467-478. CrossRef Medline

Rust NC, Schultz SR, Movshon JA (2002) A reciprocal relationship between reliability and responsiveness in developing visual cortical neurons. J Neurosci 22:10519-10523. Medline

Sireteanu R, Encke I, Bachert I (2005) Saliency and context play a role in infants' texture segmentation. Vision Res 45:2161-2176. CrossRef Medline

Swindale NV (1998) Orientation tuning curves: empirical description and estimation of parameters. Biol Cybern 78:45-56. CrossRef Medline

Tao X, Zhang B, Smith EL 3rd, Nishimoto S, Ohzawa I, Chino YM (2012) Local sensitivity to stimulus orientation and spatial frequency within the receptive fields of neurons in visual area 2 of macaque monkeys. J Neurophysiol 107:1094-1110. CrossRef Medline

Willmore BD, Prenger RJ, Gallant JL (2010) Neural representation of natural images in visual area V2. J Neurosci 30:2102-2114. CrossRef Medline

Wong-Riley M (1979) Columnar cortico-cortical interconnections within the visual system of the squirrel and macaque monkeys. Brain Res 162: 201-217. CrossRef Medline

Zhang B, Zheng J, Watanabe I, Maruko I, Bi H, Smith EL 3rd, Chino Y (2005) Delayed maturation of receptive field center/surround mechanisms in V2. Proc Natl Acad Sci U S A 102:5862-5867. CrossRef Medline

Zhang B, Smith EL 3rd, Chino YM (2008) Postnatal development of onset transient responses in macaque V1 AND V2 neurons. J Neurophysiol 100:1476-1487. CrossRef Medline

Zheng J, Zhang B, Bi H, Maruko I, Watanabe I, Nakatsuka C, Smith EL 3rd, Chino YM (2007) Development of temporal response properties and contrast sensitivity of V1 and V2 neurons in macaque monkeys. J Neurophysiol 97:3905-3916. CrossRef Medline 